\title{
Article
}

\section{Insights from echocardiography, magnetic resonance imaging, and microcomputed tomography relative to the mid-myocardial left ventricular echogenic zone.}

Agger, P, Stephenson, RS, Dobrzynski, H, Atkinson, A, laizzo, PA, Anderson, RH, Jarvis, JC, Allan, SL, Partridge, JB, Zhao, J, Zhang, H and Maclver, DH

Available at http://clok.uclan.ac.uk/16481/

Agger, P, Stephenson, RS, Dobrzynski, H, Atkinson, A, Iaizzo, PA, Anderson, $R H$, Jarvis, JC, Allan, SL, Partridge, JB et al (2016) Insights from echocardiography, magnetic resonance imaging, and microcomputed tomography relative to the mid-myocardial left ventricular echogenic zone. Echocardiography, 33 (10). pp. 1546-1556. ISSN 0742-2822

It is advisable to refer to the publisher's version if you intend to cite from the work.

For more information about UCLan's research in this area go to http://www.uclan.ac.uk/researchgroups/ and search for <name of research Group>.

For information about Research generally at UCLan please go to http://www.uclan.ac.uk/research/

All outputs in CLoK are protected by Intellectual Property Rights law, including Copyright law. Copyright, IPR and Moral Rights for the works on this site are retained by the individual authors and/or other copyright owners. Terms and conditions for use of this material are defined in the policies page. 


\title{
Insights from echocardiography, magnetic resonance imaging, and microcomputed tomography relative to the mid-myocardial left ventricular echogenic zone
}

\author{
Peter Agger M.D. ${ }^{1}$ | Robert S. Stephenson B.Sc. Ph.D. ${ }^{2,3}$ | Halina Dobrzynski Ph.D. ${ }^{4}$ | \\ Andrew Atkinson M.Phil. ${ }^{4}$ | Paul A. laizzo Ph.D., F.H.R.S. ${ }^{5}$ | \\ Robert H. Anderson M.D., Ph.D., F.R.C.Path. ${ }^{6,7}$ | Jonathan C. Jarvis B.Sc., Ph.D. ${ }^{2}$ | \\ Sarah L. Allan B.Sc., B.S.E. ${ }^{8}$ | John B. Partridge M.B.B.S., F.R.C.R., F.R.C.P., F.R.A.C.R. ${ }^{9}$ | \\ Jichao Zhao Ph.D. ${ }^{10}$ | Henggui Zhang Ph.D. ${ }^{11}$ | \\ David H. Maclver M.B.B.S., M.D. T(M), F.R.C.P., F.E.S.C. ${ }^{8,11}$
}

\author{
${ }^{1}$ Department of Cardiothoracic and \\ Vascular Surgery, Deptartment of Clinical \\ Medicine, Aarhus University Hospital, \\ Aarhus, Denmark \\ ${ }^{2}$ Research Institute for Sport and Exercise \\ Sciences, Liverpool John Moores University, \\ Liverpool, United Kingdom \\ ${ }^{3}$ School of Dentistry, The University of \\ Central Lancashire, Preston, United Kingdom \\ ${ }^{4}$ School of Medicine, University of \\ Manchester, Manchester, United Kingdom \\ ${ }^{5}$ Institute for Engineering in Medicine, \\ Department of Surgery, University of \\ Minnesota, Minneapolis, Minnesota \\ ${ }^{6}$ Institute of Genetic Medicine, Newcastle \\ University, Newcastle Upon Tyne, United \\ Kingdom \\ ${ }^{7}$ Division of Biomedical Sciences, University \\ College London, London, United Kingdom \\ ${ }^{8}$ Department of Cardiology, Taunton \\ \& Somerset Hospital, Taunton, United \\ Kingdom \\ ${ }^{9}$ Eurobodalla Unit, Rural Clinical School of \\ the ANU College of Medicine, Biology \& \\ Environment, Batemans Bay, NSW, Australia \\ ${ }^{10}$ Auckland Bioengineering Institute, \\ University of Auckland, Auckland, New \\ Zealand \\ ${ }^{11}$ Biological Physics Group, School of \\ Astronomy and Physics, University of \\ Manchester, Manchester, United Kingdom
}

Correspondence

David H. Maclver, M.B.B.S., MD., T(M)., FRCP., FESC., Department of Cardiology, Musgrove Park Hospital, Taunton, UK. Email: david.maciver@tst.nhs.uk
Background: The anatomical substrate for the mid-mural ventricular hyperechogenic zone remains uncertain, but it may represent no more than ultrasound reflected from cardiomyocytes orientated orthogonally to the ultrasonic beam. We sought to ascertain the relationship between the echogenic zone and the orientation of the cardiomyocytes.

Methods: We used 3D echocardiography, diffusion tensor imaging, and microcomputed tomography to analyze the location and orientation of cardiomyocytes within the echogenic zone.

Results: We demonstrated that visualization of the echogenic zone is dependent on the position of the transducer and is most clearly seen from the apical window. Diffusion tensor imaging and microcomputed tomography show that the echogenic zone seen from the apical window corresponds to the position of the circumferentially orientated cardiomyocytes. An oblique band seen in the parasternal view relates to cardiomyocytes orientated orthogonally to the ultrasonic beam.

Conclusions: The mid-mural ventricular hyperechogenic zone represents reflected ultrasound from cardiomyocytes aligned orthogonal to the ultrasonic beam. The echogenic zone does not represent a space, a connective tissue sheet, a boundary between ascending and descending limbs of a hypothetical helical ventricular myocardial band, nor an abrupt change in cardiomyocyte orientation.

\section{KEYWORDS}

cardiomyocyte orientation, computed tomography, diffusion tensor magnetic resonance imaging, echocardiography, helical ventricular myocardial band 


\section{1 | INTRODUCTION}

A mid-mural hyperechogenic zone within the muscular ventricular septum was initially described by Feigenbaum in 1981 in a patient with a hypertrophied left ventricle. ${ }^{1}$ At that time, the phenomenon was described as an "echo of unknown origin." For several decades thereafter, the nature of the echogenic zone remained unresolved, gaining very little scientific attention. In 2005, however, Boettler and associates studied 30 healthy humans using standard echocardiography and proposed two main hypotheses for the origin of the echogenic zone. ${ }^{2}$ Demonstrating an echogenic zone in all subjects examined, they suggested that either an abrupt change in cardiomyocyte direction, or the presence of coronary arteries in the middle of the septum, may explain the hyperechogenic properties of the area. As the echogenic zone was present in both $\mathrm{B}$ - and $\mathrm{M}$-mode images, and noting that it was best visualized in four-chamber views, they considered the ventricular septum a bilayered structure. ${ }^{2}$

A recent study in Echocardiography has now suggested that the echogenic zone may represent a boundary line between ascending and descending segments of the putative helical ventricular myocardial band ${ }^{3}$ originally proposed by Torrent-Guasp. ${ }^{4}$ Torrent-Guasp had dissected the ventricular mass following prolonged boiling of the heart. Using his dissections, and following alleged cleavage planes, he was able to produce a single band of muscle running from the pulmonary to the aortic roots. When reformed, it twisted into a rope-like structure with a helical arrangement. ${ }^{4,5}$ As has already been pointed out by Boettler and colleagues, the dissection performed by Torrent-Guasp disrupts the interconnected arrangement of the cardiomyocytes and creates artificial cleavage planes that provide the spurious impression of a bilayered ventricular septum. ${ }^{2}$

We have, in several recent scientific contributions, pointed toward the misleading nature of the concept of the helical ventricular myocardial band. It is our opinion that the notion of the myocardial band, in which the orientation of myocytes follows the long axis of this hypothetical structure from pulmonary to aortic root, is not supported by any independent anatomical studies. ${ }^{6-9}$ The conclusions of Hayabuchi and colleagues prompted us to engage in correspondence relating to their account. In our letter, we suggested that the echogenic zone, rather than representing a thin boundary between parts of the ventricular myocardial band, could be a consequence of a higher reflectivity of ultrasound from previously recognized aggregates of cardiomyocytes orientated in circumferential fashion and having an orthogonal direction to the ultrasound beam. ${ }^{10}$

It has long been recognized that, in the midwall of the left ventricle, the cardiomyocytes are aligned so as to encircle the ventricular cavity and approach a perpendicular orientation relative to the left ventricular long axis. ${ }^{8,11-13}$ These circumferentially orientated myocytes have a long axis at right angles to the ultrasonic beam when viewed from the cardiac apex in a four-chamber view. The echogenic zone, therefore, should be present in the entire circumference of the left ventricle. It is well worth noting that, to date, no imaging modalities other than ultrasound have demonstrated the presence of any well-defined anatomical structure in the left ventricular midwall in the position of the echogenic zone.

Three-dimensional echocardiography allows one to exploit the differences in any particular planar view when it is reconstructed either from a dataset obtained from an apical transducer position or from the parasternal window. When the beam is delivered from the parasternal window, all the cardiomyocytes encountered in the mural segment have similar orientation to the beam, as the principal orientation of each aggregate of cells is in or near to the tangential plane, irrespective of its helical angle. When the beam is delivered from the apex, however, only the circumferentially oriented cells in the midzone will be perpendicular to angle of incidence. The helical angle of the inner and outer zones will progressively take the orientation of the cardiomyocytes away from the perpendicular. If orientation of the cardiomyocytes to the beam is a major factor in its reflectance, then images reconstructed from parasternal insonation will show relatively even texture of reflected echoes, but those reconstructed from the apical view will be expected to show greater reflectance in the midzone. This mid-zone accentuation, moreover, would be expected to be visible in any reconstruction from apical insonation, as it is a phenomenon related to the delivery of the beam, and not to the process of reconstruction. To investigate the hypothesis that the mid-mural ventricular hyperechogenic zone arises from the circumferentially orientated cardiomyocytes, we studied the presence and extent of the echogenic zone in three-dimensional reconstructed echocardiographic views. We then compared the images with the predominant orientation of cardiomyocytes derived from both high-resolution microcomputed tomography and diffusion tensor magnetic resonance imaging.

\section{2 | MATERIALS AND METHODS}

\section{1 | Echocardiography}

Illustrative clinical echocardiographic images were obtained in a 73-year-old asymptomatic hypertensive woman using GE Vivid E9 equipment (GE Healthcare, Copenhagen, Denmark) equipped with Vivid E9 BT 11 M5S and 4V transducers. Left ventricular short-axis slices were reconstructed from $3 D$ images obtained from both the apical and parasternal windows using EchoPAC software analysis (EchoPac PC 110.1.2 software for Vivid 7 and Vivid E9, GE Healthcare).

\section{2 | Diffusion tensor imaging}

Five neonate lambs were intubated after establishment of an intravenous access and administration of Propofol $\left(1-5 \mathrm{mg} \cdot \mathrm{kg}^{-1} \cdot \mathrm{h}^{-1}\right)$ and Fentanyl (2-4 $\left.\mu \mathrm{g} \cdot \mathrm{kg}^{-1} \cdot \mathrm{h}^{-1}\right)$ by continuous infusion. The animals were ventilated (Servo300 ventilator, Siemens, Mississauga, ON, Canada) aiming at a $\mathrm{PaCO}_{2}$ between 35 and $50 \mathrm{~mm} \mathrm{Hg}$. The lambs were sacrificed with an overdose $\left(100 \mathrm{mg} \cdot \mathrm{kg}^{-1}\right)$ of thiopental sodium, and the hearts were harvested through a median sternotomy. The coronary arteries were flushed with cardioplegic solution $\left(20 \mathrm{mmol} \mathrm{L}^{-1}\right.$ 
potassium supplemented Ringer's Lactate) and subsequently fixed with $10 \%$ formalin administered manually slowly at subphysiological pressure. The hearts were stored in formalin for at least 2 days and then rinsed and stored in phosphate-buffered solution at $4^{\circ} \mathrm{C}$ until scanning.

Diffusion tensor magnetic resonance imaging was performed on all ovine hearts using a 9.4T preclinical MRI scanner (Agilent, Santa Clara, CA, USA), equipped with a $400 \mathrm{mT}$ gradients and the "vnmrJ 4.0" operating system. In the scanner room, temperature was kept constant at $22.0 \pm 1.5^{\circ} \mathrm{C}$ and air humidity at $50 \pm 10 \%$. A diffusion weighted multislice 2D spin-Echo sequence was employed with the following parameters: Isotropic voxel resolution of 310 microns, repetition time: 7000 milliseconds, echo time: 30 milliseconds. Diffusion-weighted images were acquired ${ }^{14}$ with 30 predefined isotropically distributed diffusion encoding directions, one averaging and diffusion sensitivity $b$-values of $1000 \mathrm{~s} / \mathrm{mm}^{2}$ and one image with a $b$-value of $0 \mathrm{~s} / \mathrm{mm}^{2}$. Total scan duration per heart was approximately 16 hours. The ovine hearts were harvested at the University at Buffalo, Buffalo NY, USA, and obtained under conditions approved by the local Laboratory Animal Care Committee.

\section{3 | Microcomputed tomography}

A fresh human heart of a 60-year-old female, who had died due to bronchogenic metastatic carcinoma, and which was deemed not viable for transplantion, was cardiopleged, recovered, and transported to the University of Minnesota via LifeSource (Minneapolis, MN, USA): all appropriate consents were obtained for donation of this specimen for research (HH074 on http://www.vhlab.umn.edu/atlas/histories/). The heart itself showed no known pathologic changes and was initially flushed with saline solution to remove residing clotted blood. Next, it was perfusion-fixed to maintain an end-diastolic state, as previously described, ${ }^{15}$ and transported to the University of Manchester: A Materials Transfer Agreement was executed and shipping methods followed the guidelines of the University's Environmental Health \& Safety Department. Then, the specimen was stained with $7.5 \% \mathrm{I}_{2} \mathrm{KI}$ for 14 days. The contrast agent was replaced at day 7 , as previously described. ${ }^{16,17}$ The heart was then rinsed with distilled water to remove excess contrast agent. To maintain the chambers in an inflated state, the heart was filled with warm agarose solution, which becomes a stable gel at room temperature. The heart was scanned using the Nikon Metris Custom Bay 225/320 kV CT scanner at the Manchester X-ray Imaging Facility (MXIF, University of Manchester). During image acquisition, 3142 radiographic projections were acquired over a $360^{\circ}$ of rotation, using an accelerating voltage of $155 \mathrm{keV}$ and a current of $55 \mu \mathrm{A}$. Total scan time was approximately 50 minutes. Data were reconstructed using filtered back-projection, resulting in tomographic image data with an isotropic voxel size of $73 \times 73 \times 73 \mu \mathrm{m}^{3}$.

\section{4 | Data analysis}

The diffusion tensor imaging raw data was imported using custommade software, ${ }^{18}$ and the diffusion tensors representing the

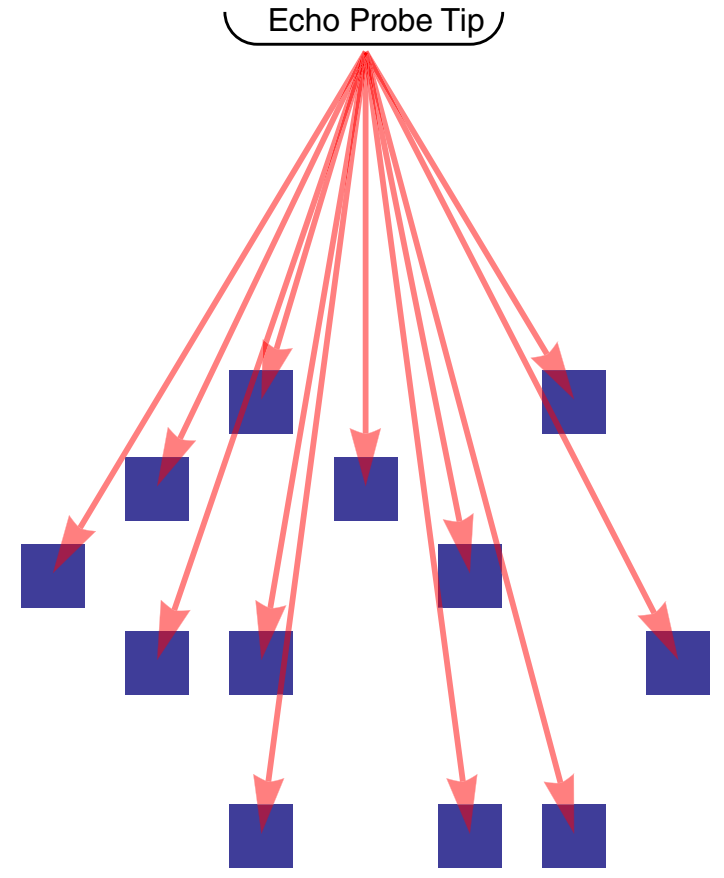

FIGURE 1 Schematic of the vector field emulating the ultra sound beam. For each voxel (blue) a vector is calculated originating from the virtual tip of the echo probe and ending in each voxel. Subsequently, the angle between the primary eigenvector within each voxel and the corresponding echo probe vector is calculated and plotted

orientation of the cardiomyocytes were calculated. Original 2D image series from microcomputed tomography were imported into MatLab Release 2011b (The MathWorks Inc., Natick, MA, USA) for further processing. Structure tensors representing the orientation of the cardiomyocytes were calculated from the raw grayscale image files as described previously using the primary eigenvector at each voxel. ${ }^{19-21}$ Subsequently, the tensor-based datasets from both modalities were imported into Mathematica (version 9, Wolfram Research Inc., Champaign, IL, USA). Appropriate short-axis and apical four-chamber views were identified to match the scan planes of conventional echocardiography. Within the planes, a fan-shaped vector field was defined mimicking the ultrasonic beam. This was done by calculating a "beam vector" for each voxel in the plane of interest, spanning from the narrow end of the virtual beam, where the scanner probe would be positioned, to the individual voxel (Fig. 1). For every voxel, the angle between the primary eigenvector of the tensor, equal to the long axis of the cardiomyocytes, and the beam vector was calculated. All angles above 75 degrees were subsequently mapped in 5-degree intervals by grayscale color coding.

\section{3 | RESULTS}

\section{1 | Echocardiography}

Conventional echocardiographic images are shown in Fig. 2. The echogenic zone is absent in most parasternal views, but present in all apical views. The zone is seen in the inferolateral and inferior regions 
FIGURE 2 Note the absence of the echogenic zone in the parasternal longaxis view (top left). The echogenic zone is easily seen in all the apical long-axis views particularly the ventricular septum, inferolateral and inferior regions (arrows) giving an apparent 3-layer structure. The left ventricle appears to have two layers in the anterolateral and anterior walls

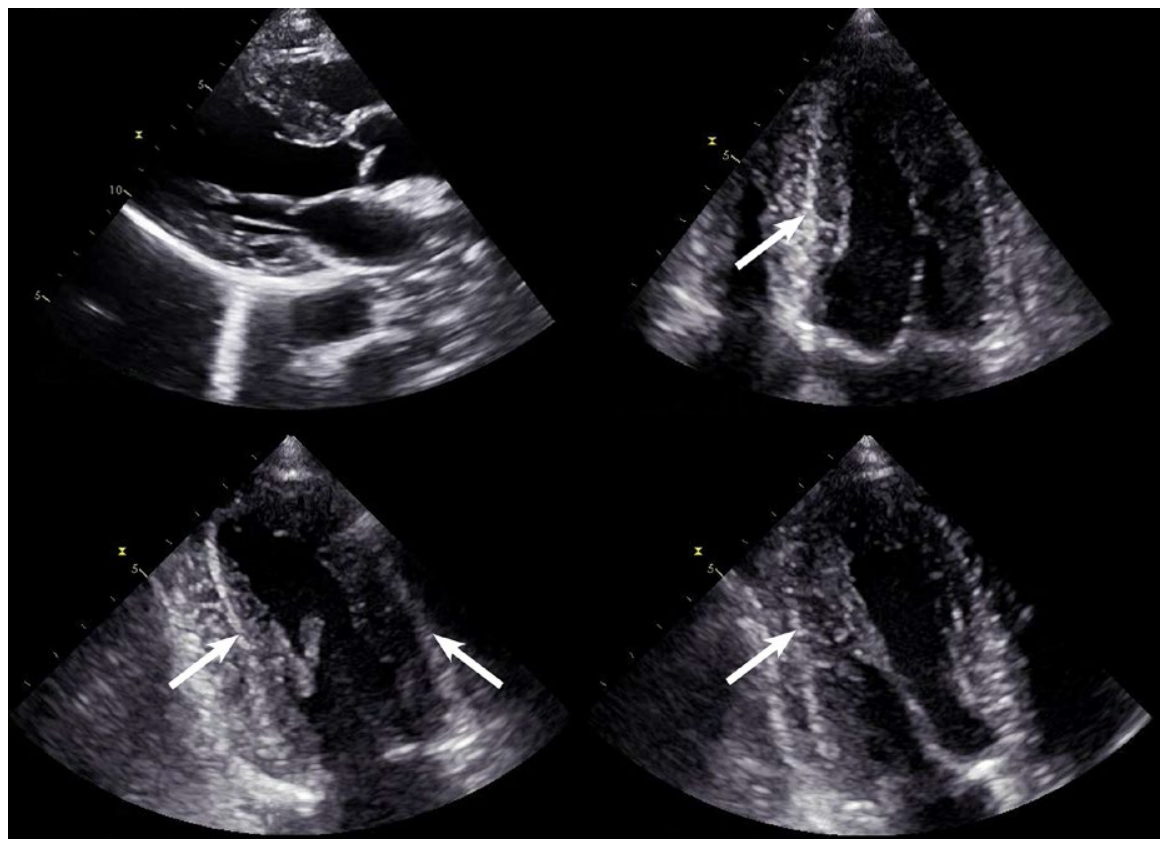

and is not confined to the septum. In Fig. 3, left ventricular short-axis images are reconstructed from three-dimensional datasets obtained from the parasternal and apical windows. In the images obtained from the parasternal window, it is evident that the echogenic zone is absent from both the original long-axis images and the reconstructed short-axis images. In contrast, in the short-axis images reconstructed from long-axis images obtained from an apical window, an obvious echogenic zone is seen in the expected mid-septal position. Here, the echogenic zone assumes the shape of a ring continuous throughout the entire left ventricle. The online supplemental movie clips of the reconstructed short-axis views, derived from the apical dataset, show that the diameter of circumferential echogenic zone decreases during systole and probably represents shortening of the midwall cardiomyocytes. An oblique echogenic zone can be found in the left ventricular free wall, when originating from the usual short-axis view from the parasternal window, but with either some angulation toward the apex or slipping more laterally between the parasternal and the apical views (Fig. 4).

\section{2 | Diffusion tensor imaging}

The results of the diffusion tensor imaging analyses are shown in Fig. 5. The location of the echogenic zone is reproduced in both the septum and left ventricular free wall when mapping the orientation of the cardiomyocytes using diffusion tensor imaging in the four-chamber view. Anatomical photographs matching the regions are provided showing no evidence of tissue planes visible to the naked eye. In the short-axis representations from the parasternal view, no echogenic zone is visible in either modality. There is a striking resemblance between the seemingly random scatter on the ultrasonic images and the gray tones in the diffusion tensor imaging dataset. The oblique echogenic zone was also replicated using diffusion tensor imaging (Fig. 4).

\section{3 | Microcomputed tomography}

The results from the cardiomyocyte orientation analyses in the human microcomputed tomographic data are compared with those of the ovine diffusion tensor data in Fig. 6. The microcomputed tomography produces a zone similar to that of echocardiography. It is most pronounced in the septum when reproduced in the equivalent of the four-chamber view. The short-axis views of the two modalities in Fig. 6, reproduced in the equivalent parasternal view, are also similar in terms of the pattern of white colors and the absence of a mid-mural ventricular hyperechogenic zone. Fig. 7 shows short-axis images reconstructed from datasets in which the angles have been calculated using the apical viewpoint. Similar to the findings in Fig. 3, the echogenic zone is present in the entire circumference of the left ventricle, as shown in the diffusion tensor data. A comparable white line is seen in the right ventricular free wall. The mid-septal zone is particularly evident in the microcomputed tomographic data, although not as clearly visible in the free walls of the left and right ventricles (Fig. 7).

\section{4 | DISCUSSION}

As far as we are aware, ours is the first study to investigate the origin of the mid-mural ventricular hyperechogenic zone of the left ventricle using a range of imaging modalities and image processing. Our investigation has combined the modalities of echocardiography, computed tomography, and magnetic resonance imaging. We have confirmed that, when using standard and three-dimensional echocardiography, the echogenic zone is best visualized in the apical views, but not well seen in the parasternal views. The diffusion tensor imaging studies, however, have shown that the position of the mid-mural hyperechogenic zones coincides with the orthogonal 


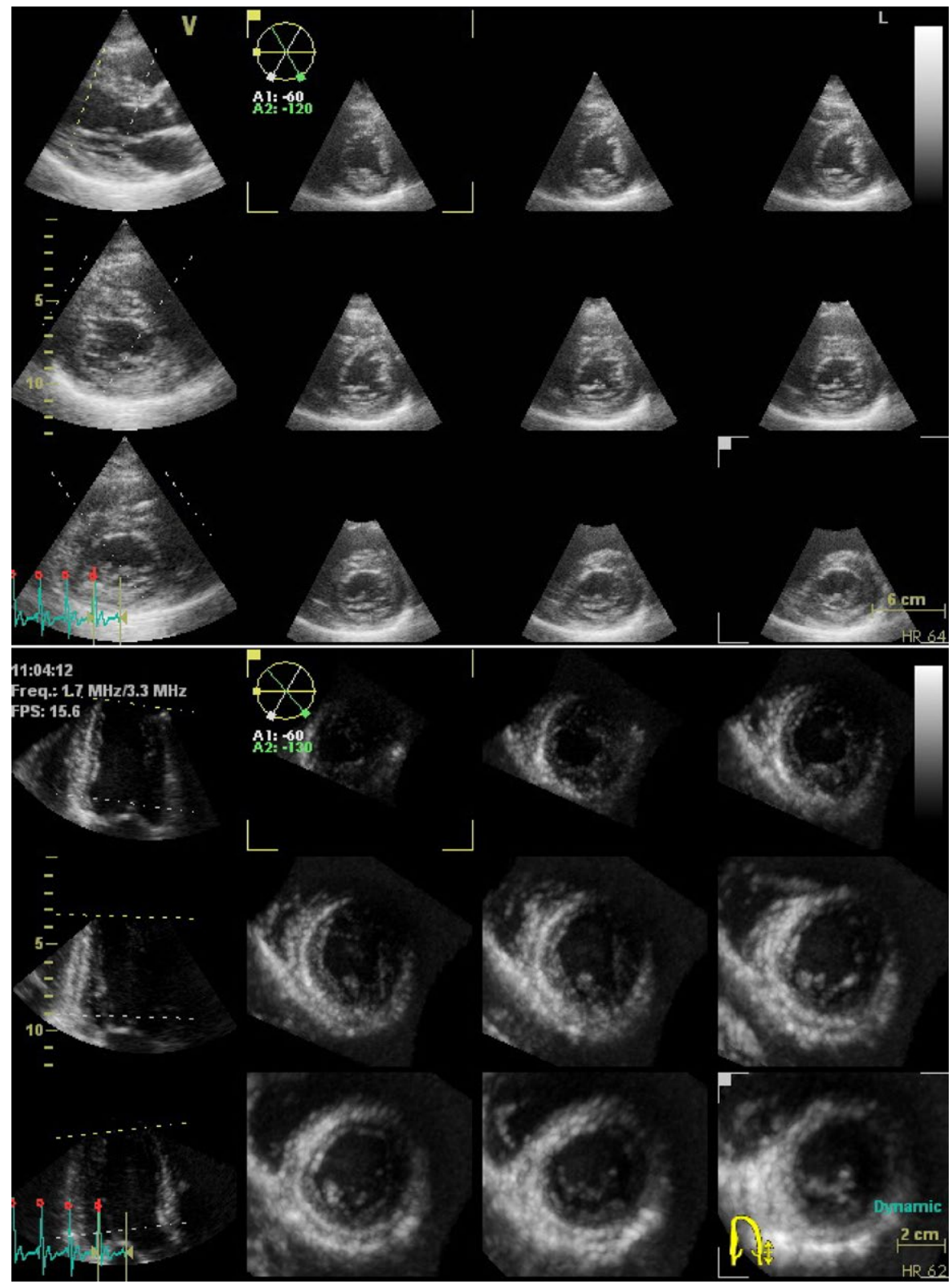

FIGURE 3 (Top) Images attained from parasternal long axis-derived images.

Note the absence of an obvious echogenic zone. (Bottom) Images attained from apical long axis-derived images. Note the presence of a near circumferential midwall echogenic zone. Left panels are the original images with dotted lines delineating the reconstruction areas. Remaining images are reconstructed slices from this area. See movie clips S1 and S2
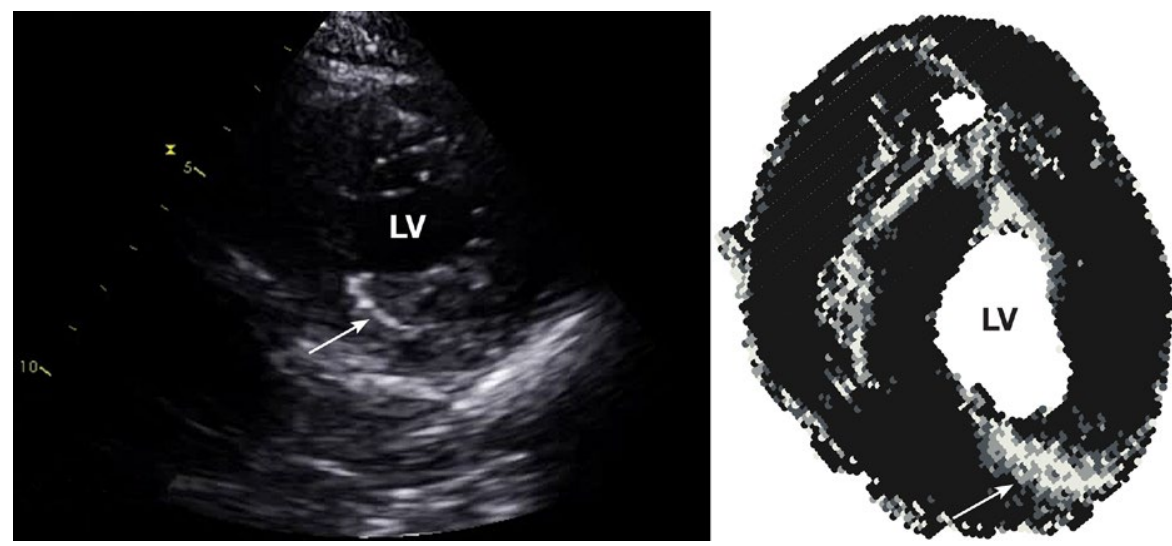

FIGURE 4 An oblique echogenic zone can sometimes be found in the left ventricular free wall, when angulating the ultrasound beam toward the apex from the parasternal window (left panel). When reproducing this viewpoint using diffusion tensor imaging, a similar oblique zone is seen (right panel)

orientation of the cardiomyocytes relative to the ultrasound beam. This latter finding, obtained using ovine hearts, was then confirmed by high-resolution microcomputed tomography of the human heart.
The oblique echogenic zone in the left ventricular free wall, shown by oblique parasternal insonation, was also reproduced by diffusion tensor magnetic resonance imaging. Taken overall, our findings are 


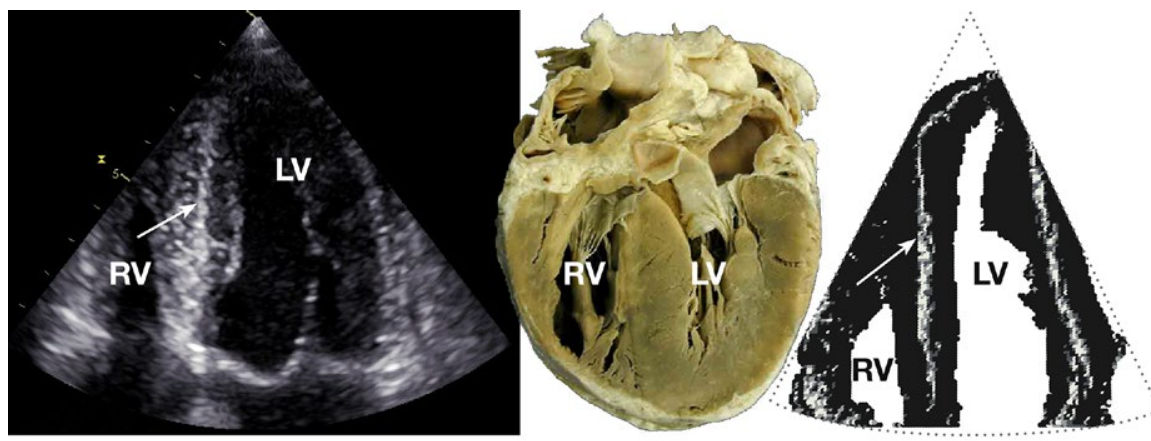

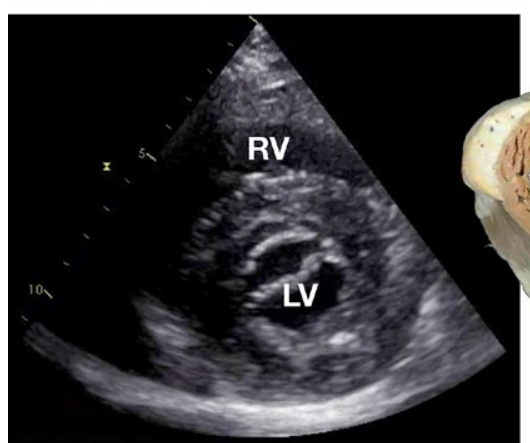

Echocardiography
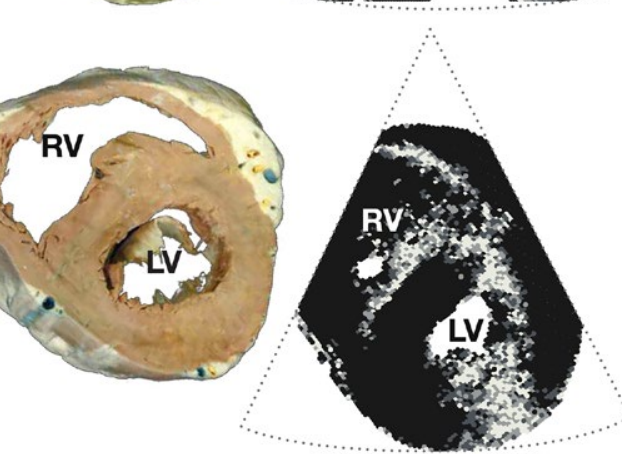

Anatomy

\section{Diffusion Tensor Imaging}

FIGURE 5 Left panels: Echocardiographic images from the apical four-chamber view (top) and the parasternal short-axis view (bottom). Note the echogenic zone in the septum in the four-chamber view (arrow) and the complete absence of any hyperechogenic areas in the shortaxis view. Center panels: Fixed human heart specimens for anatomical comparison. No macroscopic structures are to be found aiding in the explanation of the hyperechogenicity of the mid-septum. Right panels: Diffusion tensor imaging representations. The four-chamber image is flipped vertically for improved comparison with the conventional apex-up four-chamber view in echocardiography. White and gray areas indicate cardiomyocytes with an orientation perpendicular to the direction of the ultrasound beam as outlined by the dotted triangle (see methods). Note the resemblance between the short-axis echocardiography and diffusion tensor images. $L V=$ left ventricle. RV= right ventricle

consistent with both histologic and pneumatographic findings of a continuum of myocardial structure, as previously demonstrated by Lunkenheimer and Niederer. $^{22}$

Discussion on the nature of the echogenic zone is currently ongoing. Boettler and colleagues ${ }^{2}$ had already considered the possibility that the purported helical ventricular myocardial band could provide an explanation for their findings. They had emphasized that the bilayered structure disclosed by blunt dissections could only be produced by disrupting the connections between the aggregates of the cardiomyocytes. ${ }^{2}$ Despite this obvious caveat, Hayabuchi and associates, in their recent echocardiographic study, considered that the very presence of the echogenic zone provided supporting evidence for the concept of the myocardial band. ${ }^{3}$ They speculated that the hyperechogeneity is caused by an abrupt change in the orientation of the cardiomyocytes, despite the fact that many earlier studies have shown that the change in transmural orientation of all the myocytes within the left ventricle occurs gradually. $8,13,23,24$

Using short-axis reconstructions from three-dimensional echocardiography, we have now shown that the echogenic zone is not confined to the septum. When viewed from the apex, it can also be found around most of circumference of the left ventricle. Other studies have previously pointed toward the appearance of the echogenic zone in other parts of the left ventricle than the septum. ${ }^{3,25}$ To the best of our knowledge, our study is the first fully to show its extent. Furthermore, we have illustrated, using diffusion tensor imaging and microcomputed tomography, that the appearance of the echogenic zone coincides with areas in the myocardium where the orientation of the cardiomyocytes is perpendicular to the direction of the ultrasonic beam. Crosby and colleagues have elegantly shown that the ultrasonic backscatter in myocardium is highly dependent upon the orientation of the myocytes. ${ }^{26}$ Their work is in keeping with our notion that the intensity of the backscatter is increased when the cardiomyocytes are perpendicular to the direction of the ultrasonic beam. ${ }^{26}$ Their results also endorsed the findings of several earlier studies of both the heart ${ }^{27,28}$ and human tendons. ${ }^{29}$ The similarity between echocardiography, and structure tensor analysis from microcomputed tomography, and diffusion tensor imaging in our study reinforces this viewpoint. As it has been known for decades that the amount of backscatter in echocardiography is dependent upon fibrous orientation in the tissue, it is surprising that so many alternative explanations of the origin of the echogenic zone have been proposed. For example, it has been stated that the echogenic zone represents a space approximately $100 \mu \mathrm{m}$ wide, which purportedly divides the septum into two functionally different halves. ${ }^{30}$ Such a space has not been demonstrated using histology, nor by any other imaging modality other than ultrasound. It is striking, furthermore, that a transmural myocardial septal biopsy does not readily fall into two pieces when removed from the heart. In our view, it is evident that the concept of the helical ventricular myocardial band is inadequate for explaining the nature of the echogenic zone. 

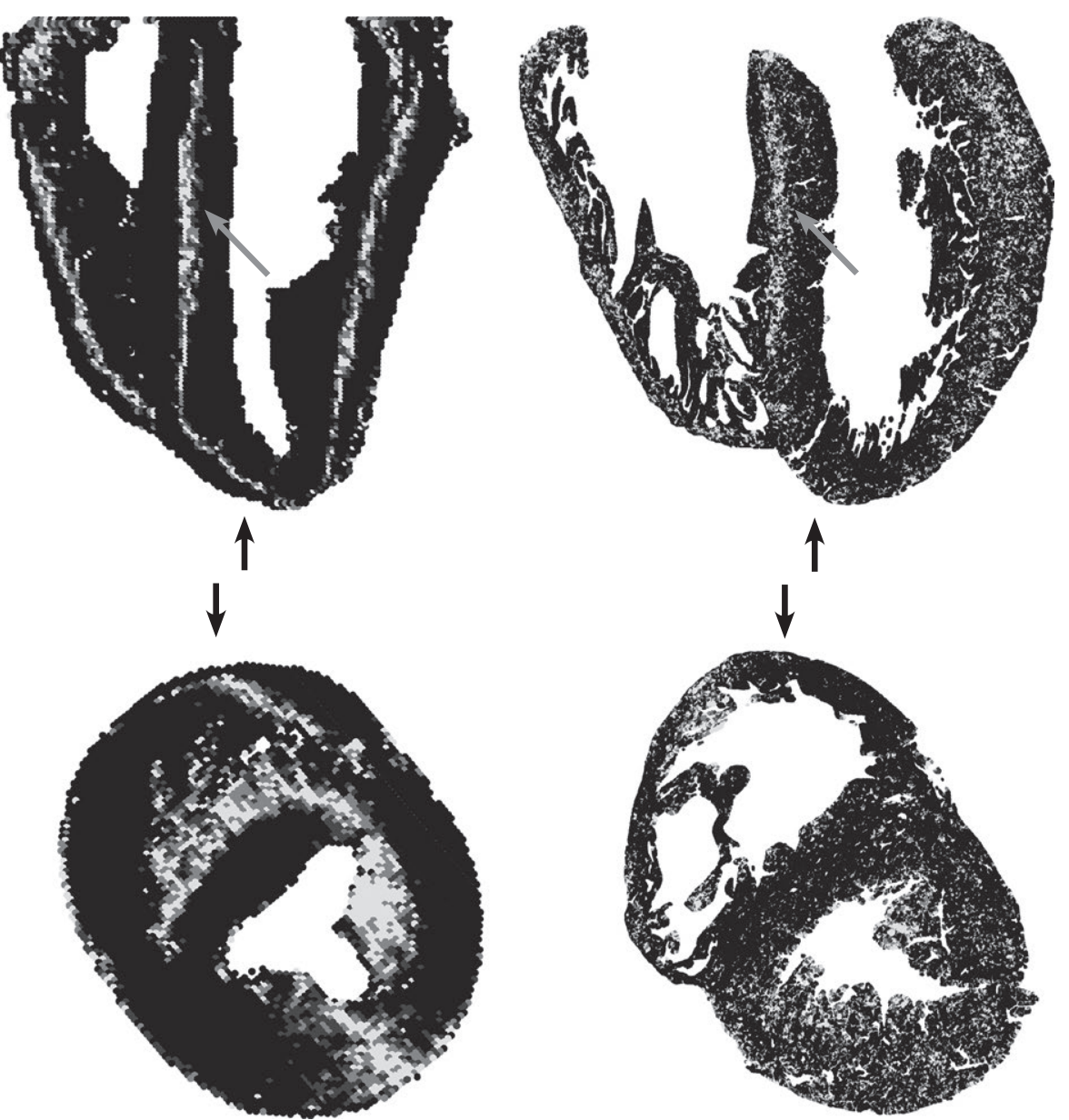

FIGURE 6 Diffusion tensor imaging (left panels) and micro-CT (right panels) representations of the conventional echocardiograpic views of the heart. White and gray areas indicate cardiomyocytes with an orientation perpendicular to the direction of the ultrasound beam (see Methods). The viewpoint of the virtual echo probe is depicted in each panel by black arrows. In the top panels, the orientation of the cardiomyocytes outlines an area similar to the echogenic zone in echocardiography (gray arrows). The zone is absent in the virtual parasternal views in both techniques (bottom panels)

\section{1 | Evidence against the helical ventricular myocardial band}

One of the main premises underscoring the notions of Torrent-Guasp ${ }^{4}$ is that the strip of oriented myocytes represented by the unwrapped heart acts like a skeletal muscle, with an origin at the pulmonary root and an insertion at the aortic root. ${ }^{31}$ It should be noted, however, that when viewing the unfolded helical ventricular myocardial band, the grain of the tissue runs in multiple directions, rather than along its length. ${ }^{32}$ In our opinion, the concept of a myocardial band is an oversimplification. It does not exist as an anatomical reality in which behavior within the band is somehow separated from behavior outside or across it. As early as 1864, Pettigrew described a myocardial continuum in seven layers of cardiomyocytes throughout the ventricular wall, showing spirals of helical grain, and a midwall layer with the myocytes oriented circumferentially. ${ }^{33}$ Based on pneumatic distension, the more recent study by Lunkenheimer and Niederer reformats Pettigrew's notion of seven layers into five approximate zones: namely subepicardial, outer, middle, inner and subendocardial zones, with the latter zone including the trabeculations. ${ }^{22}$ The zones described are of an arbitrary nature, without abrupt transition from one zone to the next, and without any discrete planes of connective tissue interposed between them. The zones, therefore, are not true layers as such, but gradual complicated regions of transition. In the short-axis view, the inner, middle and outer zones make up the bulk of myocardium. In some regions, they form multiple chevron-like shapes (Fig. 8), giving an appearance similar to a feather, and representing arrays of aggregated cardiomyocytes. ${ }^{6}$ The central zone of the left ventricular wall consists of cardiomyocytes orientated in a circumferential direction and is made up of the apices of the chevrons. ${ }^{12}$ Within this arrangement is a continuous mesh of cardiomyocytes supported by an extracellular matrix, the latter made up mostly of a collagen framework. The pneumatographic studies of Lunkenheimer and colleagues showed similar chevron-like structures, revealing how major aggregates of cardiomyocytes are separated by extracellular matrix, thus forming fan-like tissue planes running in the outer and inner zones along each wing of the chevron (Fig. 8). Between these major tissue planes are interconnecting cardiomyocytes. In the mid-zone, the cardiomyocytes merge into a circumferential orientation (Fig. 8). ${ }^{22,34}$ The concept of the helical ventricular myocardial band does not allow for this circumferential orientation of the cardiomyocytes in the ventricular midwall. The existence of such circumferentially oriented midwall myocytes, nonetheless, has been an undisputed fact for decades, $8,11-13,35,36$ and it is surprising that the proponents of the unique myocardial band ignored all these previous accounts and failed to show any evidence to contradict them. The position of the echogenic zone confirms that the signal originates from uniformly oriented circumferential cardiomyocytes, rather than from a distinct space or tissue interface between helical aggregates. 
Support for the concept of the myocardial band is also lacking from studies investigating the development of the embryonic cardiac loop. ${ }^{37,38}$ Neither does data from ventricular activation support the notion of a myocardial band. The propagation of electrical waves
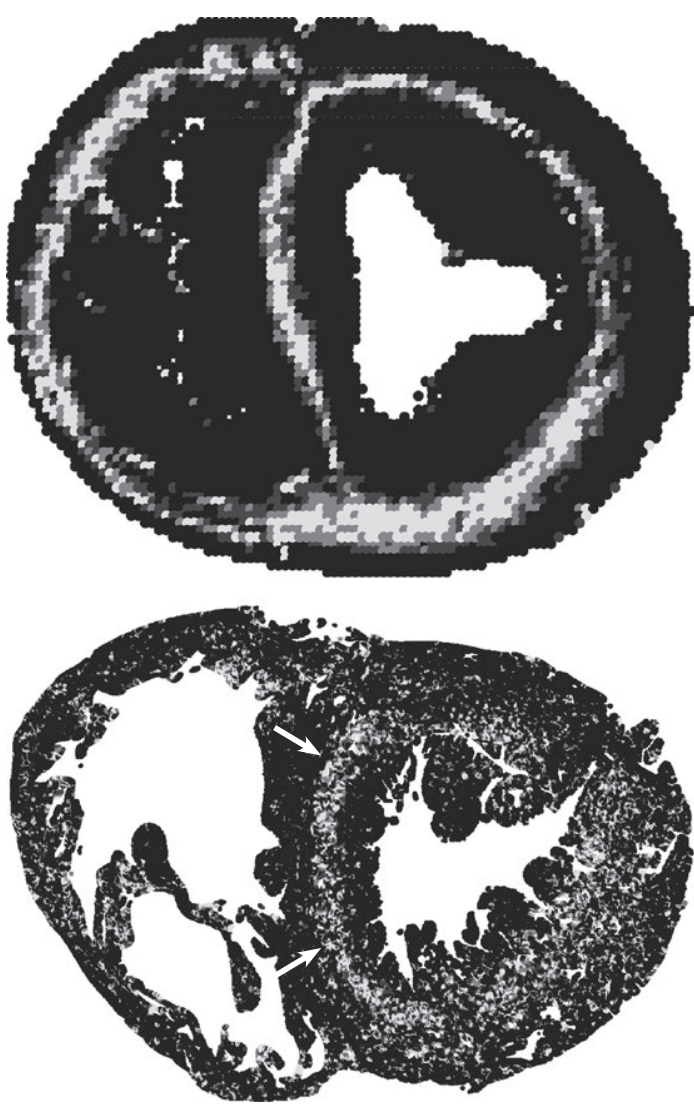

FIGURE 7 Short-axis reconstructions of diffusion tensor imaging (top) and micro-CT data (bottom). White and gray areas indicate cardiomyocytes with an orientation perpendicular to the direction of the ultrasound beam (see Methods). Angles assessed relative to the apical viewpoint are thus comparable to the bottom image in Fig. 3. Arrows mark the septal echogenic zone within the ventricles normally originates in the endocardium near the apical septum and proceeds from endocardium to the epicardium and toward the base. ${ }^{39}$ The progression of the wave front of depolarization is not consistent with activation along the alleged myocardial band, although it may well explain the delayed activation of different left ventricular segments recently found by Hayabuchi and colleagues. ${ }^{3}$ Taken overall, therefore, there is no independent anatomical evidence of which we are aware to support the concept of a single helical ventricular myocardial band. Our findings do not provide support for the notion of connective tissue planes between its alleged segments, a space between them, nor an abrupt change in the orientation of the cardiomyocytes within the ventricular midwall. The dissection of the heat-denatured bovine heart as performed by Torrent-Guasp, in our opinion, was the consequence of producing an apparent cleavage plane by entering the open side of the chevrons, splitting the apex of the chevrons to separate their inner and outer wings, and thus destroying the midwall circumferential cardiomyocyte aggregates.

\subsection{Clinical implications and structure-function relationships}

Our findings have a crucial impact in understanding the relationship between left ventricular structure and function. The motion of the left ventricle is a consequence of cardiomyocytic shortening and thickening. During systole, the epicardium shows only slight inward motion (Fig. 8), while the base moves toward the apex. ${ }^{40}$ There is little reduction in myocardial volume during systole, and therefore the wall thickens in a radial direction during contraction, displacing luminal volume, and ejecting the stroke volume. ${ }^{41}$ Cardiomyocyte shortening of approximately one-fifth results in an ejection fraction of around $65 \%$, given a normal left ventricular mural thickness. ${ }^{42}$ Absolute mural thickening is a major determinant of ejection fraction (Fig. 8). ${ }^{41}$ Any increase in mural thickness, including hypertrophy or infiltration, leads to a relative increase in absolute wall thickening and ejection fraction for a given myocardial contractile strain. ${ }^{42,43}$ Because a reduced contractile strain is seen almost universally in thick-walled ventricles, the ejection fraction is

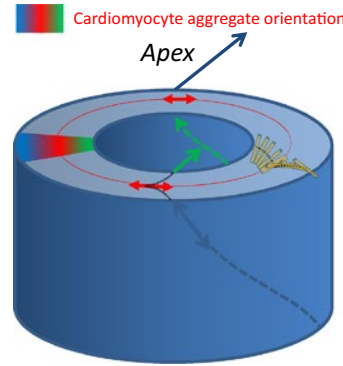

Base
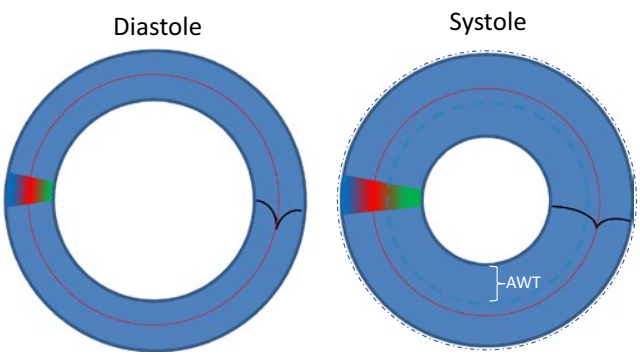

View from the apex

FIGURE 8 Multiple chevrons form a feather-like appearance with a gradual change in the dominant orientation of cardiomyocyte aggregates (left panel). The change in aggregate orientation is illustrated by the color gradient in each image. In diastole (middle panel), the apex of the chevron represents the circumferential orientated cardiomyocytes. During systole, the wall thickens flattening the chevrons (right panel). There is a minor reduction in external dimension of the left ventricle during systole (dash-dot line). The difference between end-diastolic wall and endsystolic wall thickness is the absolute wall thickening (AWT, between dashed line and endocardial border) and is an important determinant of ejection fraction. Note the subendocardium thickens to a greater extent than the subendocardium during systole 
often normal or only mildly reduced. ${ }^{44,45}$ Approximately two-thirds of the ejection fraction is attributable to midwall circumferential shortening and one-third to longitudinal shortening. ${ }^{40}$ The three-dimensional arrangement of cardiomyocytes and extracellular matrix must allow the actin-myosin interaction with cell shortening, shear strain, reorientation, sliding between aggregates to minimize shear stress, compression and thickening of long chains of cardiomyocytes, accompanied by torsion of the base relative to the apex during contraction, and a reversal of this process during relaxation (Fig. 8).

Any interpretation of cardiodynamics needs to be consistent with continuum mechanics. Inroads are now being made such that the ejection fraction can be mathematically derived from myocardial strain and wall thickness. ${ }^{46}$ Normal and abnormal left ventricular mechanics, such as twist, as well as systolic and diastolic function, furthermore, can readily be explained without invoking the concept of a helical ventricular myocardial band. ${ }^{43,47}$ The twist of the left ventricle is explained by the known anatomical arrangements of cardiomyocytes. ${ }^{43}$ Abnormalities of calcium homeostasis and collagen deposition may both result in abnormal relaxation and compliance. ${ }^{47}$ Reduced ventricular suction during early diastole is related to the combination of higher diastolic calcium levels, ${ }^{47}$ and a loss of compression energy stored during systole resulting in reduced elastic recoil. ${ }^{48}$ Abnormalities of left ventricular structure have important and quantifiable effects on myocardial function. ${ }^{49-51}$ A proper understanding of myocardial function has vital implications for comprehending the processes of cardiovascular disease such as heart failure ${ }^{52,53}$ and can be achieved without resort to a hypothetical helical ventricular myocardial band.

\section{3 | Limitations}

For the diffusion tensor imaging studies, we used ovine neonatal hearts. In contrast to human specimens, ovine hearts are readily available and small enough to fit inside the small bore of the high-field magnet. The anatomical differences between sheep and humans have not been investigated scientifically using diffusion tensor imaging, but when comparing studies of sheep ${ }^{54}$ and humans, ${ }^{23}$ the differences are small. To support this viewpoint, we conducted identical analyses using human microcomputed tomographic data and found very similar results to those obtained using the ovine hearts.

The technique of diffusion tensor imaging possesses the intrinsic limitation of averaging data. Because the myocardium is subdivided into numerous voxels each with a volume of 30 nanoliters, within each voxel the average orientation of approximately 900 cardiomyocytes is assessed. We note that the myocardium is a very heterogeneous mesh, and thus such averaging could potentially skew the data toward an unnatural degree of order. To obviate this difficulty, we also provided high-resolution computed tomographic data. In the computed tomographic dataset, each voxel contains only approximately nine myocytes; hence, smoothing is less of an issue. This higher resolution probably explains why the data obtained using computed tomography are apparently "noisier" than those provided by diffusion tensor imaging. It may also be argued that structure tensor analysis of the computed tomography data is not as accurate as diffusion tensor analysis, but this remains to be investigated. The overall consistency between all three used modalities, nonetheless, indicates that the above limitations are of minor significance.

\section{5 | CONCLUSION}

We have shown that the mid-mural ventricular hyperechogenic zone is anatomically related to the well-recognized circumferentially orientated cardiomyocytes and that the oblique echogenic zone arises from orthogonally orientated cardiomyocytes. We suggest that both echogenic zones arise as a consequence of increased reflectivity of structures such as cardiomyocytes lying perpendicular to the ultrasound beam. Our conclusions are supported by new threedimensional data from echocardiography, diffusion tensor imaging, and microcomputed tomography, as well as a wealth of previous anatomical studies. The echogenic zone does not represent an interface between the segments of a hypothetical helical ventricular myocardial band. It is, however, consistent with an intricate mesh-like structure of the myocardium.

\section{ACKNOWLEDGMENTS}

The sheep hearts used in this study were kindly provided by Prof. Robin Steinhorn, UC Davis Children's Hospital, Sacramento, CA USA, and Prof. Satyan Lakshminrushimha and Dr. Sylvia Gugino, State University of New York at Buffalo, NY, USA. We acknowledge the Danish Children Heart Foundation for the support of PA and Alder Hey Children's NHS foundation Trust for their kind support of RSS.

\section{REFERENCES}

1. Feigenbaum H: Echocardiography (3rd Edn.) Philadelphia: Lea \& Febiger; 1981; 402451:188-221.

2. Boettler P, Claus P, Herbots L, et al. New aspects of the ventricular septum and its function: an echocardiographic study. Heart. 2005;91:1343-1348.

3. Hayabuchi Y, Sakata M, Kagami S. Assessment of the helical ventricular myocardial band using standard echocardiography. Echocardiography. 2015;32:310-318.

4. Torrent-Guasp F. Anatomia Funciónal del Corazón. La actividad ventricular diastólica y sistólica. Madrid: Paz Montalvo; 1957.

5. Torrent-Guasp FF, Whimster WF, Redmann K. A silicone rubber mould of the heart. Technol Health Care. 1997;5:13-20.

6. Stephenson RS, Agger P, Lunkenheimer PP, et al. The functional architecture of skeletal compared to cardiac musculature: myocyte orientation, lamellar unit morphology, and the helical ventricular myocardial band. Clin Anat. 2016;29:316-332.

7. Anderson RH, Smerup M, Sanchez-Quintana $D$, et al. The threedimensional arrangement of the myocytes in the ventricular walls. Clin Anat. 2009;22:64-76.

8. Smerup M, Nielsen E, Agger P, et al. The three-dimensional arrangement of the myocytes aggregated together within the mammalian ventricular myocardium. Anat Rec (Hoboken). 2009;292:1-11.

9. Lunkenheimer PP, Redmann K, Niederer P, et al. Models versus established knowledge in describing the functional morphology of the ventricular myocardium. Heart Fail Clin. 2008;4:273-288. 
10. Anderson RH, Lunkenheimer PP, Jarvis JJ, et al. Assessment of the helical ventricular myocardial band using standard echocardiography. Echocardiography. 2015;32:1601-1602.

11. Feneis H. Das gefüge des herzmuskels bei systole und diastole. Morphologisches Jahrbuch. 1943;89:371-406.

12. Krehl L. Kenntniss der fillung und entleerung des herzens. Abhand Math Phys KI Koniglichen Saechs Ges Wiss 29. 1891;341:362.

13. Greenbaum RA, Ho SY, Gibson DG, et al. Left ventricular fibre architecture in man. Br Heart J. 1981;45:248-263.

14. Jones DK. The effect of gradient sampling schemes on measures derived from diffusion tensor MRI: a Monte Carlo study†. Magn Reson Med. 2004;51:807-815.

15. Eggen MD, Swingen CM, laizzo PA. Ex vivo diffusion tensor MRI of human hearts: relative effects of specimen decomposition. Magn Reson Med. 2012;67:1703-1709.

16. Stephenson RS, Boyett MR, Hart G, et al. Contrast enhanced microcomputed tomography resolves the 3-dimensional morphology of the cardiac conduction system in mammalian hearts. PLoS ONE. 2012;7:e35299.

17. Jarvis JC, Stephenson R. Studying the microanatomy of the heart in three dimensions: a practical update. Front Pediatr. 2013;1:26.

18. Agger $\mathrm{P}$, Lass $\mathrm{T}$, Smerup $\mathrm{M}$, et al. Optimal preservation of porcine cardiac tissue prior to diffusion tensor magnetic resonance imaging. J Anat. 2015;227:695-701.

19. $\mathrm{Ni} \mathrm{H}$, Castro SJ, Stephenson RS, et al. Extracting myofibre orientation from micro-CT images: An optimisation study. Computing in Cardiology Conference (CinC), 2013: IEEE; 2013, p. 823-826.

20. Zhao J, Butters TD, Zhang $\mathrm{H}$, et al. An image-based model of atrial muscular architecture: effects of structural anisotropy on electrical activation. Circ Arrhythm Electrophysiol. 2012;5:361-370.

21. Jarvis JC, Stephenson R. Studying the microanatomy of the heart in three dimensions: a practical update. Front Pediatr. 2013;1:26.

22. Lunkenheimer PP, Niederer P. Hierarchy and inhomogeneity in the systematic structure of the mammalian myocardium: towards a comprehensive view of cardiodynamics. Technol Health Care. 2012;20:423-434.

23. Toussaint N, Stoeck CT, Schaeffter T, et al. In vivo human cardiac fibre architecture estimation using shape-based diffusion tensor processing. Med Image Anal. 2013;17:1243-1255.

24. Streeter DD Jr, Spotnitz HM, Patel DP, et al. Fiber orientation in the canine left ventricle during diastole and systole. Circ Res. 1969;24:339-347.

25. Sengupta PP, Krishnamoorthy VK, Korinek J, et al. Left ventricular form and function revisited: applied translational science to cardiovascular ultrasound imaging. J Am Soc Echocardiogr. 2007;20:539-551.

26. Crosby J, Hergum T, Remme EW, et al. The effect of including myocardial anisotropy in simulated ultrasound images of the heart. IEEE Trans Ultrason Ferroelectr Frea Control. 2009;56:326-333.

27. Mottley JG, Miller J. Anisotropy of the ultrasonic backscatter of myocardial tissue: I. Theory and measurements invitro. J Acoust Soc Am. 1988;83:755-761.

28. Yang M, Krueger TM, Holland MR, et al. Anisotropy of the backscatter coefficient of formalin-fixed ovine myocardium. J Acoust Soc Am. 2007;122:581-586.

29. Hoffmeister BK, Wong AK, Verdonk ED, et al. Comparison of the anisotropy of apparent integrated ultrasonic backscatter from fixed human tendon and fixed human myocardium. J Acoust Soc Am. 1995;97:1307-1313.

30. Buckberg G, Mahajan A, Saleh S, et al. Structure and function relationships of the helical ventricular myocardial band. J Thorac Cardiovasc Surg. 2008;136:578-589.

31. Torrent-Guasp F, Ballester M, Buckberg GD, et al. Spatial orientation of the ventricular muscle band: physiologic contribution and surgical implications. J Thorac Cardiovasc Surg. 2001;122:389-392.
32. Lunkenheimer PP, Redmann K, Westermann P, et al. The myocardium and its fibrous matrix working in concert as a spatially netted mesh: a critical review of the purported tertiary structure of the ventricular mass. Eur J Cardiothorac Surg. 2006;29:S41-S49.

33. Pettigrew JB. On the arrangement of the muscular fibres in the ventricles of the vertebrate heart, with physiological remarks. Philos Trans $R$ Soc Lond. 1864;154:445-500.

34. Lunkenheimer PP, Niederer P, Sanchez-Quintana D, et al. Models of ventricular structure and function reviewed for clinical cardiologists. J Cardiovasc Transl Res. 2013;6:176-186.

35. Sanchez-Quintana D, Garcia-Martinez V, Hurle JM. Myocardial fiber architecture in the human heart. Anatomical demonstration of modifications in the normal pattern of ventricular fiber architecture in a malformed adult specimen. Acta Anat (Basel). 1990;138:352-358.

36. Ferreira PF, Kilner PJ, McGill L-A, et al. In vivo cardiovascular magnetic resonance diffusion tensor imaging shows evidence of abnormal myocardial laminar orientations and mobility in hypertrophic cardiomyopathy. J Cardiovasc Magn Reson. 2014;16:87.

37. Corno AF, Kocica MJ. Potential implications of the helical heart in congenital heart defects. Seminars in Thoracic and Cardiovascular Surgery: Pediatric Cardiac Surgery Annual: Elsevier; 2007, p. 61-67.

38. Manner J. Ontogenetic development of the helical heart: concepts and facts. Eur J Cardiothorac Surg. 2006;29(Suppl 1):S69-S74.

39. Durrer D, van Dam RT, Freud GE, et al. Total excitation of the isolated human heart. Circulation. 1970;41:899-912.

40. Maclver DH. The relative impact of circumferential and longitudinal shortening on left ventricular ejection fraction and stroke volume. Exp Clin Cardiol. 2012;17:5-11.

41. Maclver DH. A new method for quantification of left ventricular systolic function using a corrected ejection fraction. Eur J Echocardiogr. 2011;12:228-234.

42. Maclver DH, Townsend M. A novel mechanism of heart failure with normal ejection fraction. Heart. 2008;94:446-449.

43. Partridge JB, Smerup MH, Petersen SE, et al. Linking left ventricular function and mural architecture: what does the clinician need to know? Heart. 2014;100:1289-1298.

44. Maclver DH, Dayer MJ. An alternative approach to understanding the pathophysiological mechanisms of chronic heart failure. Int J Cardiol. 2012;154:102-110.

45. Maclver DH, ClarkAL. Contractile dysfunction in sarcomeric hypertrophic cardiomyopathy. J Card Fail. 2016; doi: 10.1016/j.cardfail.2016.03.020 [Epub ahead of print].

46. Maclver DH, Adeniran I, Zhang $\mathrm{H}$. Left ventricular ejection fraction is determined by both global myocardial strain and wall thickness. IJC Heart \& Vasc. 2015;7:113-118.

47. Adeniran I, Maclver DH, Hancox JC, et al. Abnormal calcium homeostasis in heart failure with preserved ejection fraction is related to both reduced contractile function and incomplete relaxation: an electromechanically detailed biophysical modelling study. Front Physiol. 2015;6:1-14.

48. Maclver DH, Dayer MJ, Harrison AJ. A general theory of acute and chronic heart failure. Int J Cardiol. 2013;165:25-34.

49. Maclver DH. Is remodeling the dominant compensatory mechanism in both chronic heart failure with preserved and reduced left ventricular ejection fraction? Basic Res Cardiol. 2010;105:227-234.

50. Smerup M, Partridge J, Agger P, et al. A mathematical model of the mechanical link between shortening of the cardiomyocytes and systolic deformation of the left ventricular myocardium. Technol Health Care. 2013;21:63-79.

51. Smerup M, Agger P, Nielsen EA, et al. Regional and epi-to endocardial differences in transmural angles of left ventricular cardiomyocytes measured in ex vivo pig hearts: functional implications. Anat Rec (Hoboken). 2013;296:1724-1734. 
52. Maclver DH. Current controversies in heart failure with a preserved ejection fraction. Future Cardiol. 2010;6:97-111.

53. Maclver DH, Clark AL. The vital role of the right ventricle in the pathogenesis of acute pulmonary edema. Am J Cardiol. 2015;115: 992-1000.

54. Walker JC, Guccione JM, Jiang Y, et al. Helical myofiber orientation after myocardial infarction and left ventricular surgical restoration in sheep. J Thorac Cardiovasc Surg. 2005;129:382-390.

How to cite this article: Agger, P., Stephenson, R. S., Dobrzynski, H., Atkinson, A., laizzo, P. A., Anderson, R. H., Jarvis, J. C., Allan, S. L, Partridge, J. B, Zhao, J., Zhang, H., Maclver, D. H. (2016), Insights from echocardiography, magnetic resonance imaging, and microcomputed tomography relative to the mid-myocardial left ventricular echogenic zone. Echocardiography, 33: 1546-1556. doi: 10.1111/echo.13324

\section{SUPPORTING INFORMATION}

Additional Supporting Information may be found online in the supporting information tab for this article.

Movie clip S1. "Band Apical SAX sweep.avi". Parasternal apical sweep. Note the presence of an echogenic zone towards the apex.

Movie clip S2. "Banded Myocardium 38 Apical.wmv". Short axis slices reconstructed from reconstructed views from three-dimensional images derived from the apical view. Note the presence of an echogenic zone.

Movie clip S3. "Banded Myocardium 36 Parasternal.wmv". Short axis slices reconstructed from reconstructed views from threedimensional images derived from the parasternal view. Note the absence of an echogenic zone. 